\title{
Abordagem Paralela para Simulação Quântica no Modelo de Máquina Geométrica Quântica
}

\author{
Murilo Schmalfuss:, Anderson Ávila ${ }^{\dagger}$ Renata Reiser, Maurício Pilla, \\ Centro de Desenvolvimento Tecnológico, UFPel, \\ 96010-610, Pelotas, RS \\ E-mail: mfschmalfuss, abdavila, reiser, pilla@inf.ufpel.edu.br
}

\author{
Adriano K. Maron \\ Department of Computer Science \\ University of Pittsburgh \\ Sennott Square, Pittsburgh/PA, USA \\ E-mail: akk48@pitt.edu.
}

RESUMO

A Computação Quântica $(C Q)$ [7] segue atingindo novos marcos rumo à construção de computadores quânticos. Apesar de todos os esforços, vários desafios técnicos limitam os sistemas atuais a alguns bits quânticos [10]. Pela indisponibilidade de hardware quântico, o estudo e desenvolvimento de aplicações na $C Q$ usualmente é feito estritamente pela especificação matemática das computações ou por meio de ferramentas de simulação. Este último caracteriza a abordagem mais prática, entretanto a complexidade computacional associada a simulação de sistemas quânticos a partir de computadores clássicos limita o tamanho dos sistemas a serem simulados.

O projeto no qual este trabalho está inserido busca consolidar a integração de vários esforços de pesquisa, com destaque para: ( $i$ ) Distribuição das computações em cluster [2]; ( $i i)$ Simulação quântica através de GPUs [5]; (iii) Simulação quântica através de CPUs multicore.

Atualmente situado sob o contexto do ambiente de simulação quântica VPE-qGM (Visual Programming Environment for the Quantum Geometric Machine Model), este trabalho tem o objetivo de estabelecer o suporte à aceleração da biblioteca de execução do ambiente através de processadores multicore, beneficiando-se dos recursos providos pela biblioteca OpenMP [3]. Consolida-se assim a biblioteca $q G M_{C}$-Analyzer, com a implementação, desenvolvimento e validação de algoritmo para simulação quântica em arquiteturas multicore, cuja modelagem foi introduzida em [9].

O ambiente VPE- $q G M$, fundamentado no modelo de processos qGM (Quantum Geometric Machine Model) [8], é constituído de construtores para modelagem e simulação gráfica de aplicações quânticas. De acordo com o modelo $q G M$, a noção de portas quânticas pode ser substituída pelo conceito de sincronização de processos elementares (PEs).

No ambiente $V P E-q G M$, o $P E$ é um elemento estruturado por três atributos: $(i) A c ̧ a \tilde{o}$ : Corresponde às transformações quânticas aplicadas a diferentes qubits em um mesmo instante de tempo; (ii) Parâmetros: Contém dados auxiliares associados à definição das transformações quânticas; (iii) Posição: Posição de escrita em um espaço de memória global e compartilhada, na qual é armazenado o resultado calculado pelo $P E$.

Neste contexto, uma transformação quântica, aplicada a $N$ qubits, pode ser modelada pela sincronização de $2^{N} P E s$, cujas parametrizações satisfazem as condições equivalentes à definição dos vetores componentes da matriz (transformação unitária ou de medida) associada [6].

*Bolsista de Iniciação Científica PIBIC/CNPq

${ }^{\dagger}$ Bolsista de PROBIC/FAPERGS 
Assim, durante a simulação, ocorre a execução (sequencial ou síncrona) dos $P E s$, os quais têm suas correspondentes computações efetuadas pela biblioteca $q G M$-Analyzer, manipulando os dados presentes nas posições de memória e simulando o comportamento de um sistema quântico.

A biblioteca de execução dos PEs, denominada qGM-Analyzer, implementa otimizações que controlam o aumento exponencial dos vetores componentes das matrizes de definição do operador de múltiplos qubits, conforme introduzido em [4]. Os resultados relacionados comprovam a redução no consumo de memória durante a simulação, suportando algoritmos com 11 qubits. Entretanto, o tempo total de simulação obtido permanece elevado, devido a quantidade de operações necessárias para simular uma transformação quântica.

A implementação da biblioteca $q G M$-Analyzer em $C++$ segue as otimizações introduzidas em [4], apenas alterando as estruturas de dados utilizados e fazendo uso de recursos nativos oferecidos pela linguagem visando a otimização da execução. Visando a compatibilidade da biblioteca com o ambiente $V P E$ - $q G M$, é considerada a biblioteca Boost 1.49 .0 [1] para integração das duas linguagens envolvidas. Para a implementação do paralelismo foi utilizada A biblioteca OpenMP [3] implementa o paralelismo.

O módulo Boost-Python auxiliou no desenvolvimento da biblioteca $q G M_{C}$-Analyzer permitindo que sejam reutilizados no código $C++$ tipos do Python, como as listas de valores utilizadas no ambiente $V P E-q G M$, e ainda realizando a conversão destes tipos para tipos da linguagem $C++$. Outra funcionalidade utilizada do módulo Boost-Python foi a geração da biblioteca compartilhada importada pelo ambiente em Python.

A implementação em $C++$ manteve o mesmo algoritmo desenvolvido em [4], porém a forma de armazenamento dos dados e os algoritmos para acesso a esses dados foram modificados para diminuir a complexidade da biblioteca. Dentre os fatores que contribuíram para um melhor desempenho, destacam-se a utilização de vetores como alternativa as listas do Python e o fato de a linguagem $\mathrm{C}++$ ser compilada, permitindo ao compilador otimizações no código gerado.

$\mathrm{Na}$ implementação paralela, cada thread possui uma cópia privada da pilha, e as memórias de escrita e leitura são compartilhadas entre todos os threads, pois cada valor calculado é escrito em uma posição diferente da memória. A divisão dos threads é feita de forma a manter juntos os valores das matrizes necessários para o cálculo de uma posição. Para tal, iterações são divididas levando em conta o número de colunas da primeira matriz envolvida na transformação. Nos casos em que a transformação possui apenas uma matriz, os threads são divididos de forma diferente, para que o trabalho seja distribuído de forma balanceada. A definição do número de threads utilizadas pela biblioteca é definida por uma variável de ambiente (OMP_NUM_THREADS), gerando uma implementação mais flexível. Ou seja, dependendo da transformação quântica, tem-se um controle da granulosidade visando melhor desempenho da biblioteca.

Para validação e análise de desempenho da implementação da $q G M$-Analyzer em $C++$, foram desenvolvidos estudos de caso envolvendo sincronizações arbitrárias de transformações quânticas, contemplando sistemas entre 13 e 20 qubits.

A metodologia dos testes utilizada contempla, para cada estudo de caso, a realização de 15 simulações. A máquina utilizada na simulação possui as seguintes características: processador Intel Core i7-3770 @ 3.4 GHz,8GB RAM e sistema operacional Ubuntu 12.04 64 bits.

A principal comparação de desempenho se dá com a execução da biblioteca em diferentes números de threads. Os testes foram realizados com transformações Hadamards, representada por uma matriz densa de ordem $2^{n}$, onde $n$ é o número de qubits e transformações Pauli $X$, uma matriz esparsa de ordem $2^{n}$. Estas execuções compreenderam duas etapas: $(i)$ Geração dos valores não nulos associados ao correspondente vetor componente da matriz de definição da transformação quântica modelada; (ii) Multiplicação desses valores pelas amplitudes obtidas da estrutura de memória que modela o espaço de estados do sistema quântico. Nas simulações das Hadamards, devido ao elevado número de operações, pode-se facilmente observar um desempenho elevado, ou seja, o aumento no número de qubits diretamente relacionado com a diminuição significativa do tempo de execução. Nas transformações controladas Pauli $X$ houve um aumento no tempo de execução. Justifica-se este fato pelo baixo número de operações en- 

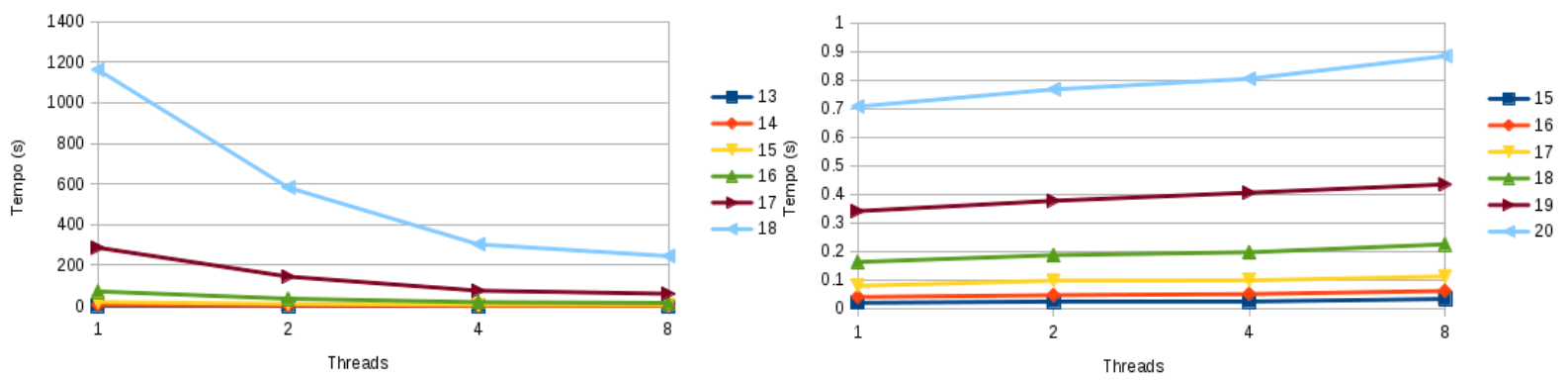

Figura 1: Tempos das transformações Hadamard e Pauli X em relação ao número de threads.

volvidas. Sendo a Pauli $X$ uma matriz esparsa, seus valores zerados não são computados pelo algoritmo otimizado, tornando o overhead da criação e troca de contexto dos threads o principal custo da transformação, como observado no decremento de desempenho da Figura da Pauli X.

Os tempos de simulação, em cada estudo de caso, abrangendo os casos para 1, 2, 4 e 8 threads são descritos na Figura 1. Como analisado, os tempos de execução para as transformações Hadamards obtiveram os melhores desempenhos. As transformações Pauli $\mathrm{X}$ tiveram o seu tempo de execução aumentando conforme aumentava o número de threads, pois envolvem poucos cálculos, e seu tempo é dominado pela criação e troca de contexto dos threads.

As otimizações realizadas e a implementação paralela representaram um ganho significativo no tempo de execução das transformações quânticas, permitindo a simulação de transformações de 18 qubits com elevado número de operações, como no caso das Hadamards.

A continuidade do trabalho consiste na integração da biblioteca $q G M_{C}$-Analyzer com o ambiente $V P E-q G M$ além da expansão para suporte a transformações multiqubits e na implementação de aplicações em outras bibliotecas disponíveis, para comparação de desempenho entre diferentes ferramentas para suporte a simulação quântica.

Palavras-chave: VPE-qGM, Computação Quântica, OpenMP, Simulação Quântica

\section{Referências}

[1] Boost, Boost 1.49.0 library documentation, www.boost.org/doc/libs/1_49_0/, (2012).

[2] A. Ávila; A. Maron; R. Reiser and M. Pilla, Extending the VirD-GM environment for the distributed execution of quantum processes, Proc. of the XIII WSCAD-WIC, (2012), 1-4.

[3] E. Ayguade and B. Chapman, "Introduction: Special Issue: OpenMP", Scientific Programming, 2003.

[4] A. Maron; A. Ávila; R. Reiser and M. Pilla, Introduzindo uma nova abordagem para simulação quântica com baixa complexidade espacial, Anais do DINCON 2011, (2011), 1-6.

[5] A. Maron; R. Reiser and M. Pilla, High-performance quantum computing simulation for the quantum geometric machine model, Proceedings of CCGRID 2013, (2013), 1-8.

[6] A. Maron; R. Reiser and M. Pilla, Quantum Processes: A Novel Optimization for Quantum Simulation, TEMA Tendências em Matemática Aplicada e Computacional], 2013 (a ser publicado).

[7] M. Nielsen and I. Chuang, "Quantum Computation and Quantum Information", Cambridge University Press, 2000.

[8] R. Reiser and R. Amaral, The quantum states space in the qGM model, Proc. of the III WECIQ, (2010) 92-101.

[9] M. Schmalfuss; A. Maron; R. Reiser and M. Pilla, qGM $_{C}$-Analyzer: biblioteca para suporte à simulação quântica em C++, Proc. of the XIII WSCAD-WIC, (2012).

[10] W. Steeb and Y. Hardy, Problems and solutions in quantum computing and quantum information, World Scientific, (2004). 